\title{
Design of the mechanical system of lower limb rehabilitation equipment rehabilitation
}

\author{
Wengang Zheng ${ }^{1, a}$, Zhiyi Zhang ${ }^{2}$ b $^{*}$ \\ ${ }^{1}$ College of Mechanica Engineering, Beihua University, Jilin Province, People's Republic of China \\ ${ }^{2}$ Engineering Training Center, Beihua University, Jilin Province, People's Republic of China \\ aemail:985304275@qq.com, bemail:zczhang6@163.com, ${ }^{\circ}$ email:@163.com
}

Key words: Correction system; Mechanism design; Physical force

Abstract:In this paper, Analysis and study of various kinds of internal and external treatment instruments of knee, Sum up the advantages and disadvantages of the same products and puts forward the design idea of X/O type leg correction bed with safe, low cost and physical external forces. Combined with the biomechanical properties of cartilage and analyzes the vibration system of rehabilitation, To mathematical modeling of the mechanical analysis, check of the structure strength, in order to ensure the rehabilitation motion security and stability of the system, and the vibration control system are designed. which makes the system more convenient and flexible. The vibration system on the market and other rehabilitation instrument of vibration system is more safe and stable, the operation is more flexible, the therapy effect of patients is more significant.

\section{Introduction}

At present, the domestic and foreign treatment of knee varus instrument is little, for lower limb disability patients can not get effective treatment, so the development of orthopedic rehabilitation instrument is particularly important, and the vibration system of rehabilitation to the key role of the whole treatment process. This paper study on the biomechanical properties of cartilage and the analysis of the vibration system of rehabilitation, the mathematical modeling of the mechanical structure, the research and analysis of its structural strength to ensure safety and stability of the vibration system of the rehabilitation. At the same time, the control system is designed, which makes the system more flexible and convenient.

\section{Mechanism and Principle of The Vibration System of Rehabilitation}

The leg vibration part includes two parts of bracket and vibration zone as shown in figure 1,After the patient received the clamp for about 40 minutes, the lower leg is placed on the vibration band, The operator makes the patient's legs dangling,according to the needs of different patients, choose different frequencies of vibration, vibration frequency will be displayed in the data display part of the display.Through vibration to accelerate the blood circulation in the legs of patients and accelerate the regeneration of cartilage tissue at the knee joint [1,2],play a role in promoting blood circulation, relieve the patient for a long time to clamp the correction caused by a little discomfort.

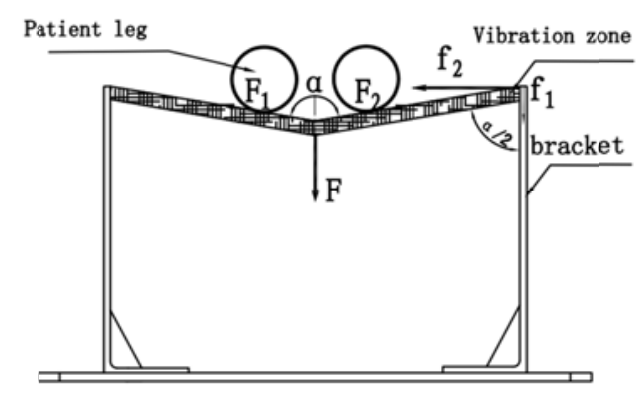

Fig. 1 Sketch of vibration mechanism 
The dynamic transmission mechanism of the leg is a low frequency, low intensity resonator, which is a crank slider mechanism. A model for the motion of its resonance is established (Figure 2).

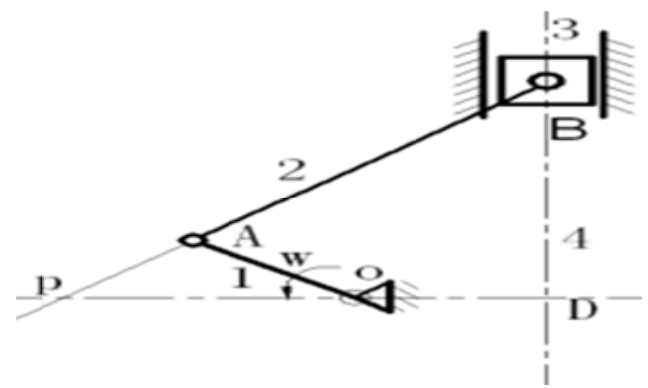

Fig. 2 The principle of vibration excitation unit schematic

Crank slider crank mechanism OAB, crank, connecting rod, slide block are respectively 1, 2, 3, the frame is a member 4 , the point $\mathrm{P}$ is the speed of the component 1 and the sliding block 3 , the component 3 is horizontal movement along the guide groove.According to the principle of instantaneous center of velocity:

$$
\overrightarrow{V_{3}}=\overrightarrow{V_{3 p}}=\overrightarrow{w_{1}} \times L_{A P}
$$

$\vec{V}_{3}$ is a member of the translation speed of $3, \vec{V}_{3 p}$ is the 3 component velocity vector point $\mathrm{P}, \vec{w}_{1}$ is a member of the angular velocity vector of $1, \overrightarrow{\mathbf{L}_{A P}}$ is the displacement vector $\mathrm{A}$ to point $\mathrm{P}$. Time differential to above formula:

$$
\frac{d \vec{V}_{3}}{d t}=\frac{d \vec{V}_{3 p}}{d t}=\frac{d \vec{w}_{1}}{d t} \times \overrightarrow{L_{A P}}+\overrightarrow{w_{1}} \times \frac{d \overrightarrow{L_{A P}}}{d t}
$$

Component 1 by the motor with uniform rotation speed,So the upper type becomes:

$$
\overrightarrow{a_{3}}=\overrightarrow{a_{3 p}}=\overrightarrow{w_{1}} \times \frac{d \overrightarrow{L_{A P}}}{d t}
$$

The velocity curve and acceleration curve (Figure 3) can be used by the formula (3). It is shown that the motion characteristics of the mechanism 3 for linear motion of variable acceleration,And the acceleration of the first positive to decrease after the reverse.Its movement law accords with the mechanical properties of the cartilage, which is consistent with the "instantaneous" elastic response of the cartilage in the loading and unloading.

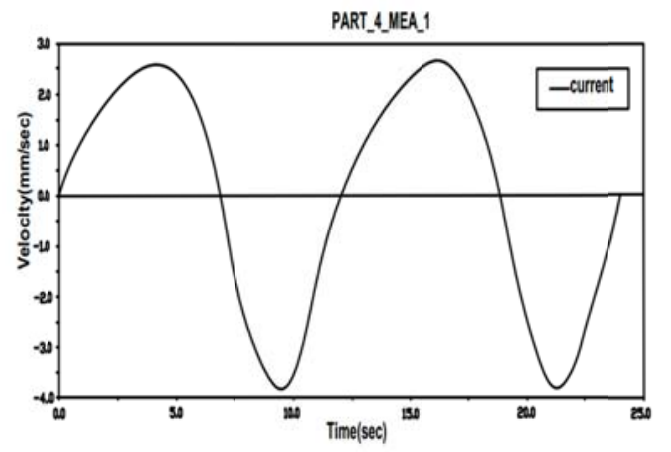

Fig. 3 Resonance velocity curve

\section{Strength Check of the Support Plate of Shattering Zone}

The vibration band length is $400 \mathrm{~mm}$, the vibration of the plate spacing is $370 \mathrm{~mm}$. The force 
analysis of the patients' legs on the belt, the vibration band is the study object[3,4],A vibration brought to bear the weight for $m$, The force of the patient to the vibration band $F=m g$, Suppose $m=\frac{1}{5} M(\mathrm{M}$ is the patient's weight).Obtained by the force equilibrium condition:

$$
F-F_{1} \cos \frac{a}{2}-F_{2} \cos \frac{a}{2}=0
$$

Take the vibration of the bearing plate as the research object to make the stress analysis chart(Figure4),By trigonometric functions:

$$
\tan \frac{\alpha}{2}=\frac{185}{\sqrt{200^{2}-185^{2}}} \approx 2.37
$$

The vibrating plate is fixed at one end and the other end is free, so it can be simplified as a cantilever beam[5,6], Its length is $l=220 \mathrm{~mm}$.the lower end of the supporting plate is fixed, the upper end is concentrated force $f_{2}$.In the distance from the board $X$ is assumed to be a branch of the branch, take the upper end plate as the research object,Moment equation by equilibrium condition:

$$
\sum M=0, f_{2} x+M=0
$$

Solution $M=-f_{2} x(0 \leq x<l)$,Absolute maximum bending moment at the bottom end of the supporting plate, The risk section is a section of the bottom end of the supporting plate, which is a rectangular cross section. And the absolute value of the maximum bending moment is: $|M|_{\max }=\frac{F l}{2} \tan \frac{\alpha}{2} \approx 49.296 \mathrm{~N} \cdot \mathrm{m}$.Dangerous section normal stress of the formula for calculating the maximum normal stress of the beam is:

$$
\begin{aligned}
& \sigma_{\max }=\frac{M_{\max }}{W_{Z}} \\
& W_{Z}=b h^{2} / 6=7.2 \times 10^{-7} \mathrm{~m}^{3}, \sigma_{\max }=68.5 \mathrm{MPa}<[\sigma]=158 \mathrm{MPa}
\end{aligned}
$$

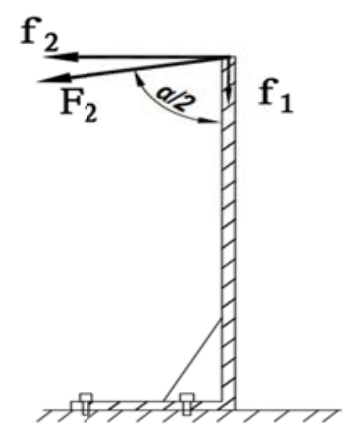

Fig. 4 Thediagram of the force of a supporting plate

Therefore, the mechanical strength of the support plate to meet the strength requirements needed for the rehabilitation instrument.The design of the supporting plate at the bottom with stiffeners, enhance the flexural performance of the board, Therefore, when the patient will be placed on the vibration of the legs, the supporting plate does not bend deformation.

\section{Vibration Frequency Detection Control Unit}

Vibration unit generally does not cause damage to patients, but if the frequency is too high may 
cause patients with leg discomfort, for patients to understand the most suitable for their own vibration frequency, so that the next use can be directly adjusted to the last frequency value.The system uses the frequency detection circuit and display circuit (Figure 5,6), the circuit structure is simple, fast response, detection accuracy, basic meet the detection requirements [7].

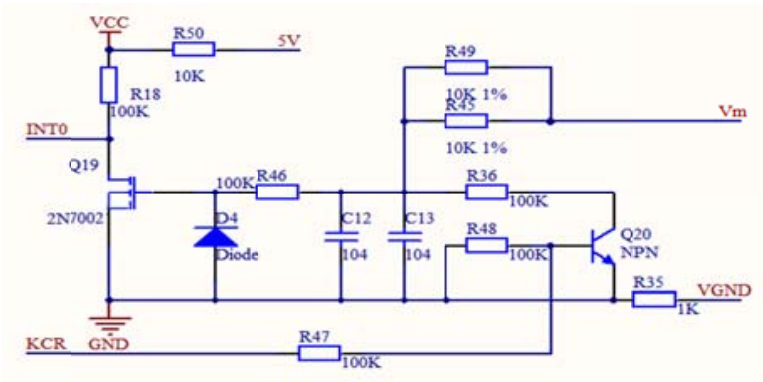

Fig. 5 Vibration frequency detection circuit

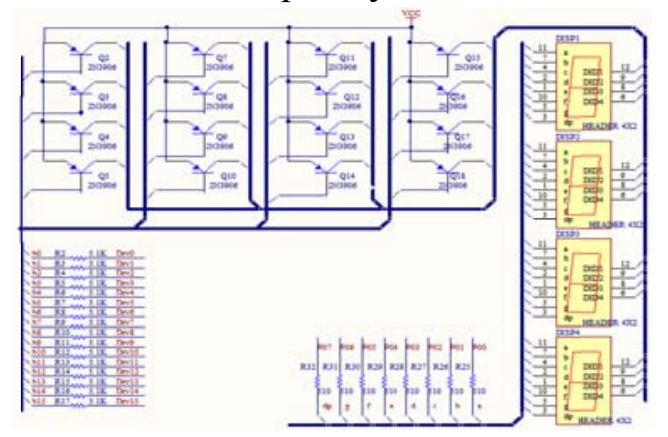

Fig. 6 Vibration frequency display circuit

\section{Conclusion}

Correction of lower extremity rehabilitation vibration system is mainly in order to correct the people with X-O type leg design, The characteristics of ergonomic design to rehabilitation is safe and effective for the biomechanics of human body based on,combined with the study on the biomechanical properties of cartilage and the analysis of the vibration system rehabilitation,Analysis of motion model mechanical resonance,check its structural strength, to ensure the safe and stable rehabilitation vibration system; Analysis of motion model mechanical resonance, check its structural strength, to ensure the safe and stable rehabilitation vibration system; control system and the vibration system are designed, which makes the system more convenient and flexible to use vibration. Compared with other market rehabilitation vibration system of the vibration system more secure and stable, the operation is more flexible, the treatment effect is more significant.

\section{References}

[1]Zhangyue.Optimization design and research of modern orthopedic rehabilitation apparatus[J]. Graduate degree thesis of Southwest Jiaotong University,2009.5.

[2]Yao Yinhua, Wang Jinhai,ZhengYu,etal.Design of multifunction medical treatment bedsystem basedon SoC single chip[J]. Journal of tianjin Polytechnic University,2006.25(4):44-47.

[3]HuMaosong.Automatic mechanical mechanism[M]. Beijing:Science and technology press, 1980.

[4]HindhedeIUffe.MachineDesignFundamentals:A Practical Approach[M]. New York:Wiley,2003.

[5]Huang Zhijian.Mechanical Design Practice[M],China Machine Press,2005. 
[6]Zhaohan.Huangkang.Chenke.Mechanical System Design[M],Higher Education Press,2004.

[7]Jiang Shengyuan,Hu Yanjuan,Wangyao,Li Jianyong.Design of intelligent multifunctional electric rehabilitation bed control system based on MCS and 51 single chip microcomputer[J]. Mechanotronics ,2008.[6]:42-45. 